\title{
Oxygen Therapy and Their Risk in The Premature Neonates: Review Article Ahmed El-Abd Ahmed Saad ${ }^{1}$, Hala M. Sakhr ${ }^{1}$, Osama Abd-Elmonem Elkady ${ }^{2}$ and Eslam Mohammed Mohammed Eltaky*1 \\ Department of ${ }^{1}$ Pediatrics and ${ }^{2}$ Ophthalmology, South Valley University, Egypt \\ *Corresponding author: Eslam Mohammed Mohammed Eltaky, Mobile: (+20)0105050546, E-mail: doctoreltaqy@gmail.com
}

\section{ABSTRACT}

Background: Neonatal respiratory care includes oxygen therapy, which is more common due to the improved survival rates of premature infants. Considering that most current studies demonstrate that preterm newborns benefit most from oxygen saturations $\left(\mathrm{SpO}_{2}\right)$ of 91-95 percent, which reduces the risk of complications such as retinopathy of prematurity, we set out to study oxygen treatment and its risks in premature neonates.

Objective: The reviews article highlight oxygen therapy and their risk in the premature neonates.

Conclusion: There have been improvements in outcomes for babies with extremely low birth weight when using a larger goal range (85\%-93\%), but these ranges have not been thoroughly assessed in major clinical trials; additional data is required. High oxygen levels may be avoided with early use of a pulmonary vasodilator.

Keywords: Hypoxia, Neonatal Resuscitation, Neonate, Oxygen, Oxygen Saturation, Retinopathy of Prematurity.

\section{INTRODUCTION}

According to the Vermont Oxford Network (VON), 95\% of extremely preterm new-born at or before 30 weeks gestation got oxygen treatment in $2016^{(1)}$. Pneumovascular and pulmonary artery hypertension are well-known outcomes of alveolar hypoxia, but bronchopulmonary dysplasia (BPD, also known as chronic lung disease of prematurity or infancy) retinopathy of prematurity (ROP) and white matter damage are less well-known ${ }^{(2)}$.

The oxygen pulse saturation is used to monitor supplementary oxygen in preterm newborns because of this $\left(\mathrm{SpO}_{2}\right)$. Low (85-89\%) or high (91-95) $\mathrm{SpO}_{2}$ goal remains open, with the low target increasing mortality (3) and the risk of necrotizing enterocolitis, while high (91-95\%) SpO2 target raises the risk of ROP (3). Currently, the American Academy of Pediatrics recommends a $\mathrm{SpO}_{2}$ goal of 90-95 percent, which is in keeping with European standards that recommend a $\mathrm{SpO}_{2}$ target of 90-94 percent ${ }^{(4)}$.

Consequences include frequent hypoxia and hyperoxia exposure, which may be harmful to the patient's health. The proportion of time spent above (>95 percent) the goal was reduced, while the percentage of time spent under (85 percent) the target was increased as a consequence of education and training of the clinical staff to enhance $\mathrm{SpO} 2$ maintenance within the target range ${ }^{(5)}$.

The objective of review article the highlight oxygen therapy and their risk in the premature neonates.

\section{Prematurity \\ Definition of preterm:}

Preterm birth refers to an infant being born before the mother has reached the full 37 weeks of her pregnancy (259 days). To reliably distinguish between preterm and term birth, precise estimates of gestational age are essential ${ }^{(6)}$.

\section{Subcategories of Preterm:}

According to gestational age, there are three types of preterm birth: Extremely preterm (before 28 weeks), very preterm (between 28 and 32 weeks), and moderate to late preterm (32 to 37 weeks). ${ }^{(7)}$.

\section{Epidemiology:}

Every year, an estimated 15 million premature births occur. The figure is a little more than one in ten newborns. Preterm birth problems claim the lives of over one million children each year. Many cancer patients will be disabled for the rest of their lives, whether it's through learning difficulties or issues with their vision or hearing. For children under the age of five, preterm is by far the largest cause of mortality worldwide. As a result, preterm birth rates are rising in almost every country with good data ${ }^{(8)}$. According to these global and regional estimates, there are significant differences between and within nations ${ }^{(6)}$. Preterm problems account for $38 \%$ of all newborn fatalities in Egypt, despite the fact that the general population's prevalence of preterm delivery is estimated at less than $10 \%{ }^{(9)}$.

\section{Causes of preterm labour:}

Infection or inflammation, uteroplacental ischaemia or hemorrhage, uterine overdistension, stress, and other immunologically driven processes are now all recognised to have a role in preterm labour syndrome (10). Factors that are related with preterm delivery, but are not clearly linked to the onset of preterm labour, have been used to explain premature labour. Risk factors are increasingly being linked to the transition 
from uterine quiescence to premature labour or preterm premature rupture of the membranes (PPROM) ${ }^{(11)}$.

Predicting preterm births by identifying risk variables is a worthwhile endeavour for a number of reasons. Identifying women who are at risk is the first step in implementing risk-specific therapy. It's also possible that a group of people with certain risk indicators may be used to evaluate certain therapies. Finally, the identification of risk variables may provide light on the processes that contribute to premature delivery (12). Maternal and foetal characteristics that have been linked to preterm birth include maternal demographics, nutritional status and pregnancy history as well as psychological characteristics, adversity, infection and uterine contractions and cervical length. There are also a number of biological and genetic markers ${ }^{(12)}$.

\section{Complications \\ Mortality:}

Mortality and morbidity in newborns due to preterm delivery is one of the leading causes. Preterm birth problems (12 percent, 1.033 million, uncertainty range [UR] 0.717 million-1.216 million) were the most common cause of neonatal mortality, accounting for 41 percent (3.575 million) of the projected 8.795 million deaths of children under the age of five in $2008^{(\mathbf{1 3})}$.

\section{Respiratory Distress Syndrome (RDS):}

Respiratory distress syndrome affects around 24,000 newborns each year, or $80 \%$ of those delivered before the $27^{\text {th }}$ week of pregnancy (RDS). Surfactant deficiency is linked to RDS ${ }^{(14)}$.

\section{Bronchopulmonary Dysplasia and Chronic Lung Disease:}

Bronchopulmonary dysplasia (BPD) is a term used to describe the chronic lung disease (CLD) that may accompany RDS in premature newborns (BPD). Chronic obstructive pulmonary disease (COPD) and chronic obstructive pulmonary disease (COPD) are two distinct but related conditions. It has been linked to difficulties with childhood growth, health, and neurodevelopment ${ }^{(15)}$.

Lung immaturity is the major cause of BPD/CLD; however additional factors such as positive-pressure ventilation, high oxygen concentration, infection, and other inflammatory triggers also contribute to this condition ${ }^{(16)}$.

\section{Importance of Diagnostic Tests for Monitoring Efficacy of Treatment for Preterm Labor:}

Neither perinatal death nor prematurity-related newborn morbidity was demonstrated to be improved by the current gold standard therapy for preterm labour, namely tocolytics ${ }^{(17)}$. Because of this, the development of novel and possibly more effective therapies for preterm labour is critical. In order to create new therapies for this condition, scientists must first develop reliable methods for detecting labour ${ }^{(18)}$.
The current approaches do not allow for the distinction between patients in "real" preterm labour, who would deliver preterm if not treated, and those in "false" preterm labour, who present with labour signs and symptoms but would not deliver preterm regardless of therapy. Preterm birth may be predicted with a positive predictive value (PPV) of only around 50\% using the approaches that are presently in use. A patient's participation in "fake" work is inevitable in investigations of therapy efficacy ${ }^{(19)}$.

\section{Oxygen therapy for premature infants:}

Patients were given oxygen based on their skin colour, as well as their breathing patterns (breathing frequency and regularity). As recently as the 1960s and 1970 's, equipment for more exact monitoring of the physiologic action of a drug was not yet available (microsampling of blood gases, transcutaneous oxygen monitoring, and subsequently pulse oximetry) ${ }^{(20)}$.

It is a primary objective of oxygen treatment that patients get sufficient oxygenation while consuming the lowest possible proportion of oxygen provided. However, a number of obstacles stand in the way of reaching this objective. Although systematic oxygen delivery in paediatrics has been practised for more than 75 years, the term "normoxia" (oxygen administration that avoids both the harmful consequences of hypoxia and the harmful effects of hyperoxia) is still vague, leading to considerable variances in practise. Adequate oxygenation is an ill-defined concept even among the medical community ${ }^{(21)}$.

\section{Assessment of Inadequate Oxygen Delivery:}

In order to determine a patient's requirement for oxygen, there are a number of physical and laboratory indicators that may be analysed. $\mathrm{A} \mathrm{PaO}_{2}$ that is lower than normal, usually $80 \mathrm{~mm} \mathrm{Hg}$, may be used to detect hypoxemia. It is common practise to provide oxygen when $\mathrm{PaO}_{2}$ falls below $60 \mathrm{~mm} \mathrm{Hg}$ in children, although this alone cannot be used to assess the appropriateness of oxygen administration (23). An ability to provide oxygen depends on the concentration of hemoglobin in the blood, the level of oxygen saturation, and the pace at which blood circulates. Oxygen supply is often phrased as follows: Blood hemoglobin $(\mathrm{Hb})$ and oxygen saturation $\left(\mathrm{SaO}_{2}\right)$ are both expressed as percentages in the equation oxygen delivery equation $\left(\mathrm{DO}_{2}\right)=\mathrm{CO}[(\mathrm{Hb}$ $\left.\left.\times \mathrm{SaO}_{2} \times 1.34\right)+\left(\mathrm{P} \mathrm{aO}_{2} \times 0.0031\right)\right]^{(20)}$.

In this equation, you can see that $\mathrm{PaO}_{2}$ is predicated on a very little quantity of dissolved oxygen in the blood. Even with a normal $\mathrm{PaO}_{2}$, oxygen supply may be insufficient in a patient who is anemic or hypovolemic, has an atypical hemoglobin with higher oxygen affinity, or has a low CO. Hypoxia is a common term to describe this situation of insufficient oxygen supply. In the next paragraphs, we'll go into further depth on hypoxia ${ }^{(22)}$. 


\section{Indications:}

Hypoxemia, or low oxygen levels in arterial blood, necessitates the use of supplemental oxygen treatment. In order to properly digest carbohydrates and produce adenosine triphosphates, oxygen is required. Tissue hypoxia occurs when oxygen levels fall short of what the body needs to operate properly ${ }^{(20)}$.

In addition to tissue necrosis and an increased risk of kernicterus, this hypoxia may also result in localised vasodilation, pulmonary vasoconstriction, and metabolic acidosis. There's a chance that hypoxia may cause damage to the brain ${ }^{(23)}$.

\section{Contraindications:}

Oxygen therapy has relatively few contraindications, although in patients with ductaldependent lesions, oxygen therapy may induce overcirculation in the pulmonary system since it is a vigorous pulmonary vasodilator (pulmonary blood vessel dilation). Retinopathy of prematurity and bronchopulmonary dysplasia may be reduced by lowering the $\mathrm{SpO}_{2}$ in preterm infants ${ }^{(24)}$.

\section{Goals of Oxygen Therapy:}

Adequate tissue oxygenation is the end objective of oxygen delivery. The patient's size and clinical condition must be taken into consideration while selecting a supplementary oxygen delivery method. As a result, the lowest feasible fractional concentration of inspired oxygen $\left(\mathrm{FIO}_{2}\right)$ must be used for oxygen treatment ${ }^{(20)}$.

Allowable hypoxemia in critically ill children has been shown to be lung-protective by providing a patient with severe disease (often mechanically ventilated) a level of oxygen delivery adequate to avoid hypoxia while minimising the detrimental effect of a toxic level of oxygen and toxic ventilator support ${ }^{(25)}$.

The classic definition of hypoxemia was extended by Martin and Grocott, who proposed terminology based on the body's physiologic reaction to the length of time it had been present. When hypoxemia occurs over a long period of time, it may impact the choice to use a permissive hypoxemic, normoxic, or supranormal cardiac output approach ${ }^{(26)}$.

\section{Oxygen Toxicity:}

Paul Bert first discovered the harmful consequences of oxygen treatment in the late 1800s when employing hyperbaric oxygen systems. Longterm exposure to a 1.0 fraction of delivered $\mathrm{O}_{2}\left(\mathrm{FDO}_{2}\right)$ level has been shown to produce chest discomfort, with symptoms resembling bronchopneumonia in some cases. Capillary endothelium is initially damaged, then interstitial edoema develops, and finally the alveolarcapillary membrane thickens after 72 hours of exposure to high oxygen concentrations. Type I alveolar cells are killed and type II cells proliferate if the process continues. Physiologic shunting and increasing hypoxemia occur as a consequence of an exudative phase ${ }^{(27)}$.

High quantities of normobaric oxygen have lately emerged as a potential danger, as well. One may expect that as a gas exchange interface to the human body, the lung would be at the highest danger of injury. Numerous research on animals and healthy human volunteers have shown this damage to exist. The sort of damage found in ARDS has been linked to this injury. Oxygen concentration and treatment time seem to have a direct effect on damage severity ${ }^{(28)}$.

Oxidative processes or radical-mediated mechanisms are the primary means by which reactive oxygen species (ROS), such as hydroxyl ion and peroxynitrite, interact with lipids, DNA, and proteins (28).

In the case of severe brain damage, normobaric hyperoxia may be beneficial. Normobaric hyperoxia (100 percent oxygen) for two hours was reported to enhance the redox status of the brain in persons with excessive brain lactate by Vilalta et al. ${ }^{(29)}$, in Spain. Hyperoxia may enhance patient outcomes in the proper patients, even if no other outcomes were evaluated. In pediatrics, more study is required to balance the various possible consequences of high oxygen concentrations on the overall fate of people with severe traumatic brain damage.

\section{During Resuscitation:}

A patient's chances of being released from the hospital even after a cardiac arrest both in and outside of the hospital remain low. Many toddlers die within a few days of spontaneous circulation returning due of neurological or cardiovascular dysfunction. Oxygen supplementation is important during and after resuscitation. It's no secret that hypoxia is associated with a worse prognosis after cardiac arrest ${ }^{(30)}$.

\section{Infants:}

Perhaps the dosage is a crucial aspect in producing better results. The use of oxygen in the delivery room for resuscitation has been linked to an increased risk of pediatric cancer, according to a little amount of data. So the American Heart Association suggests starting resuscitation with room air or blended oxygen in preterm babies and then titrating $\mathrm{FIO}_{2}$ to acceptable $\mathrm{SpO}_{2}$ levels as a result of this information ${ }^{(31)}$.

\section{Oxygen effects on premature infants:}

Antioxidant protection from plasma radical scavengers such as beta-carotene, antioxidant enzymes such as glutathione peroxidase, and a lack of superoxide dismutase in preterm newborns make them sensitive to oxidative stress. In hyperoxia and re-oxygenation following hypoxemia, toxic oxygen radicals are elevated ${ }^{(32)}$.

\section{Retinopathy of prematurity:}

It is thought that retinal immaturity and low retinal arterial oxygenation are more major causes of ROP than 
any other single factor. Premature neonates with retinal degeneration may have a rise in blood pressure and hyperoxia. Hypoxia, increased vascular endothelial growth factor, and a proliferation of new arteries and fibrous tissue that invades the vitreous are all possible consequences. Fibrous tissue contraction may lead to retinal detachment ${ }^{(33)}$.

\section{Monitoring of Oxygen Therapy:}

After the needed assessment as detailed before, it is important to monitor the patient's reaction to therapy and the intended physiologic outcome. This technique comes with a way to measure its success. The majority of doctors employ noninvasive monitoring methods like pulse oximetry, arterial blood gases, or partial pressure of oxygen $\left(\mathrm{PaO}_{2}\right)$ to help in their clinical assessment ${ }^{(34)}$.

Pre-pulse oximetry, you could tell whether your $\mathrm{SpO}_{2}$ level was between 80 and 85 percent by looking at your skin colour. Intermittent, invasive arterial blood samples may also be used to assess $\mathrm{pH}, \mathrm{PaCO}_{2}$, and $\mathrm{PaO}_{2}$. Second, you'd check to see whether the patient's attempt to breathe or degree of distress improved after administering oxygen. Pulse oximetry has made it simple, noninvasive, and somewhat accurate to determine arterial oxygen saturation in clinical practise (34).

It has since been proven by McCulloh et al. ${ }^{(35)}$, that the use of intermittent pulse-oximetry does not reduce the length of stay for non-hypoxemic infants and young children who are hospitalised for the treatment of bronchiolitis, nor is it associated with any difference in escalation of care or use of diagnostic or therapeutic measures. There was no definitive resolution to the issue of whether hypoxemic patients receiving oxygen treatment could undergo this procedure, but it did provide the framework for future research.

\section{CONCLUSION}

As one of the most often used therapies in neonatology, oxygen has long been debated over the ideal oxygen saturation levels for premature neonates. A lengthy history of oxygen utilisation in this population has shown that liberal oxygen supply is associated with ROP and BPD, whereas oxygen limitation increases newborn mortality and neurological impairment.

A pulse oximeter $\left(\mathrm{SpO}_{2}\right)$, a bedside device that measures the patient's $\mathrm{FIO}_{2}$ level, is the current standard of care for continuous monitoring. The intrinsic pulmonary immaturity, the requirement of respiratory support therapies, and challenges connected to the bedside caregivers' abilities to manage $\mathrm{FIO}_{2}$ have made it difficult to attain this goal consistently in clinical practise, despite studies favouring this method.

Despite several studies, no ideal oxygen saturation target range for premature babies has been discovered. In both the neonatal critical care unit (NICU) and for premature neonates, this is true. However, those who are vulnerable should be shielded against exposure to oxygen levels that are too high or too low.

\section{Declarations:}

Consent for Publication: I confirm that all authors accept the manuscript for submission.

Availability of data and material: Available. Competing interests: None.

Funding: No fund.

Conflicts of Interest: The authors declare no conflicts of interest regarding the publication of this paper.

\section{REFERENCES}

1. Kennedy K, Cotten C, Watterberg K et al. (2016): Prevention and management of bronchopulmonary dysplasia: Lessons learned from the neonatal research network. Seminars in Perinatology, 40(6): 348-355.

2. Cherian $S$ (2013): Oxygen therapy in preterm infants. http://dx.doi.org/10.1016/j.prrv.2012.12.003

3. Manja V, Lakshminrusimha S, Cook D (2015): Oxygen saturation target range forextremely preterm infants: a systematic review and meta-analysis. JAMA Pediatr., 169:332-340.

4. Sweet D, Carnielli V, Greisen G et al. (2017): European consensus guidelines on themanagement of respiratory distress syndrome - 2016 update. Neonatology, 111:107-125

5. Poets C, Roberts R, Schimdt B et al. (2015): Association between in term it tent hypoxie mia and bradycardia and late death ordisability inextremely preterminfants. JAMA., 314: 595-603.

6. Frey H, Klebanoff M (2016): The epidemiology, etiology, and costs of preterm birth. Seminars in Fetal and Neonatal Medicine, 21(2): 68-73.

7. Quinn J, Munoz F, Gonik B et al. (2016): Preterm birth: Case definition \& guidelines for data collection, analysis, and presentation of immunisation safety data. Vaccine, 34(49): 6047-6056.

8. Vogel J, Chawanpaiboon S, Moller A et al. (2018): The global epidemiology of preterm birth. Best Practice \& Research Clinical Obstetrics \& Gynaecology, 52: 312.

9. Chawanpaiboon S, Vogel J, Moller A et al. (2019): Global, regional, and national estimates of levels of preterm birth in 2014: a systematic review and modelling analysis. The Lancet Global Health, 7(1): 37-46.

10. Boyle A, Rinaldi S, Norman J et al. (2017): Preterm birth: Inflammation, fetal injury and treatment strategies. Journal of Reproductive Immunology, 119: 62-66.

11. Meller C, Carducci M, Ceriani J et al. (2018): Preterm premature rupture of membranes. Arch Argent Pediatr., 116(4): 575-581.

12. Oskovi Z, Ozgu-Erdinc A (2018): Prediction of preterm birth: maternal characteristics, ultrasound markers, and biomarkers: an updated overview. Journal of Pregnancy, 18: 8367571.

13. Ray J, Park A, Fell D (2017): Mortality in infants affected by preterm birth and severe small-forgestational age birth weight. Pediatrics, 140(6): 20171881.

14. Saboute M, Kashaki M, Bordbar A et al. (2015): The incidence of respiratory distress syndrome among 
preterm infants admitted to neonatal intensive care unit: a retrospective study. Open Journal of Pediatrics, 5(04): 285-88.

15. Tracy M, Berkelhamer S (2019): Bronchopulmonary dysplasia and pulmonary outcomes of prematurity. Pediatric Annals, 48(4): 148-153.

16. Davidson L, Berkelhamer S (2017): Bronchopulmonary dysplasia: chronic lung disease of infancy and long-term pulmonary outcomes. Journal of Clinical Medicine, 6(1): 4-8.

17. Younger J, Reitman E, Gallos G (2017): Tocolysis: Present and future treatment options. Seminars in Perinatology, 41: 493-504.

18. Garfield L, Chin E (2020): Pharmacology for preterm labor. The Journal of Perinatal \& Neonatal Nursing, 34(2): 155-161.

19. Di Renzo G, Cabero Roura L, Facchinetti F et al. (2017): Preterm labor and birth management: recommendations from the European Association of Perinatal Medicine. The Journal of Maternal-Fetal \& Neonatal Medicine, 30(17): 2011-2030.

20. Walsh B, Smallwood C (2017): Pediatric oxygen therapy: a review and update. Respiratory Care, 62(6): 645-661.

21. Perrone S, Bracciali C, Di Virgilio N et al. (2017): Oxygen use in neonatal care: a two-edged sword. Frontiers in Pediatrics, 4: 143-48.

22. Connor D (2019): Blood Gas Analysis. Maths, Physics and Clinical Measurement for Anaesthesia and Intensive Care. Columbia University - Law Library. Pp. 97-112. https://www.cambridge.org/core/terms

23. Marcelo C, Susana R, García-Alix A et al. (2021): Hypoxic Ischemic Encephalopathy in Units Reporting to the Ibero-American Society of Neonatology Network: Prevalence and Mortality. MEDICC Review, 23(1): 3035 .

24. Sarkar P, Rahman M (2018): Oxygen Therapy in Children-An Update. Dhaka Shishu (Children) Hospital Journal, 34(1): 48-54.

25. Ketcham S, Sedhai Y, Miller $\mathbf{H}$ et al. (2020): Causes and characteristics of death in patients with acute hypoxemic respiratory failure and acute respiratory distress syndrome: a retrospective cohort study. Critical Care, 24(1): 1-9.

26. Martin D, Grocott M (2013): Oxygen therapy in critical illness: precise control of arterial oxygenation and permissive hypoxemia. Critical Care Medicine, 41(2): 423-432.

27. Bert P, Hitchcock M, Hitchcock F (1943): College Book Company, F.C. Long; Columbus, Ohio: 1943. Barometric Pressure. Researches in Experimental Physiology. Columbus: College Book Company, Pp. 1055. https://www.worldcat.org/title/barometricpressure-researches-in-experimentalphysiology/oclc/1041064869

28. Thomson L, Paton J (2014): Oxygen toxicity. Paediatric Respiratory Reviews, 15(2): 120-123.

29. Vilalta A, Sahuquillo J, Merino M et al. (2011): Normobaric hyperoxia in traumatic brain injury: does brain metabolic state influence the response to hyperoxic challenge?. Journal of Neurotrauma, 28(7): 1139-1148.

30. Bhanji F, Topjian A, Nadkarni V et al. (2017): Survival rates following pediatric in-hospital cardiac arrests during nights and weekends. JAMA Pediatrics, 171(1): 39-45.

31. Wyckoff M, Aziz K, Escobedo M et al. (2015): Part 13: neonatal resuscitation: 2015 American Heart Association guidelines update for cardiopulmonary resuscitation and emergency cardiovascular care. Circulation, 132(18): 543-560.

32. Moore T, Ahmad I, Zimmerman M (2018): Oxidative stress and preterm birth: an integrative review. Biological Research for Nursing, 20(5): 497-512.

33. Bashinsky A (2017): Retinopathy of prematurity. North Carolina Medical Journal, 78(2): 124-128.

34. Lellouche F, L'Her $\mathbf{E}$ (2020): Usual and advanced monitoring in patients receiving oxygen therapy. Respiratory Care, 65(10): 1591-1600.

35. McCulloh R, Koster M, Ralston S et al. (2015): Use of intermittent vs continuous pulse oximetry for nonhypoxemic infants and young children hospitalized for bronchiolitis: a randomized clinical trial. JAMA Pediatrics, 169(10): 898-904. 